\title{
The paramedian supracerebellar-transtentorial approach to remove a posterior fusiform gyrus arteriovenous malformation
}

\author{
Mehmet Volkan Harput, MD, and Uğur Türe, MD \\ Department of Neurosurgery, Yeditepe University School of Medicine, İstanbul, Turkey
}

This is the case of a 14-year-old female who presented with headache and seizures. Cranial magnetic resonance imaging revealed an arteriovenous malformation (AVM) located at the posterior portion of the right-sided fusiform gyrus. Cerebral angiography showed that the AVM was fed mainly by branches from the inferior temporal trunk of the posterior cerebral artery. The main venous drainage was to the right transverse sinus through the tentorial vein. The AVM was totally excised through the paramedian supracerebellar-transtentorial approach with the patient in a semisitting position. Postoperative MRI and cerebral angiography confirmed the total resection. The patient was discharged on the 5th postoperative day without neurological deficit.

The video can be found here: https://youtu.be/QPrUI8AP7G8.

KEYWORDS AVM; arteriovenous malformation; fusiform gyrus; paramedian supracerebellar-transtentorial approach; PST approach 